\title{
MRI/US fusion prostate biopsy: Our initial experience
}

\author{
Vito Lacetera ${ }^{1}$, Bernardo Cervelli ${ }^{1}$, Antonio Cicetti ${ }^{1}$, Giuliana Gabrielloni ${ }^{1}$, Michele Montesi ${ }^{1}$, \\ Roberto Morcellini ${ }^{1}$, Gianni Parri ${ }^{1}$, Emilio Recanatini ${ }^{1}$, Gianluca Giglioni ${ }^{2}$, \\ Andrea Benedetto Galosi ${ }^{2}$, Valerio Beatrici ${ }^{1}$ \\ ${ }^{1}$ Azienda Ospedaliera Ospedali Riuniti Marche Nord, Pesaro, Italy; \\ ${ }^{2}$ Clinica Urologica, Università Politecnica delle Marche, Azienda Ospedaliera Ospedali Runiti, Ancona, Italy.
}

\begin{abstract}
Summary Aim: The objective of this study is to present our initial experience with magnetic resonance imaging/ultrasound (MRI/US) fusion biopsy using the Koelis Trinity device after the first consecutive 59 patients.

Materials and methods: 59 consecutive patients with suspected prostate cancer (PCA) underwent prostate biopsy using Trinity Koelis ${ }^{\circledR}$ (Koelis, Grenoble, France). We divided the patients into 2 groups: patients with a previous negative mapping underwent to a MRI/US fusion re-biopsy (Group A); and biopsy-naïve patients who underwent to a first stereotactic 3-D mapping of the prostate (Group B). Group A (22 patients):mean age 64 years (CI 48-73), mean PSA = 7.7 $\mathrm{ng} / \mathrm{ml}$ (CI 4.2- 9.9); mean prostate volume $55 \mathrm{ml}(\mathrm{CI}$ 45-82), Digital Rectal Examination (DRE) positive in 2/22, number of lesions detected by MRI 1.4, mean cores from each MRI target lesion 3 (CI 2-5), mean total cores 15 ( CI 12-19). Group B (37 patients): mean age 66 years (CI 49-77), mean $P S A=4.7$ (3.2- 7.9); mean prostate volume $45 \mathrm{ml}$ (33-67), DRE positive in 5/37, mean total cores 14 ( CI 10-16) Results: In Group A 10/22 patients were positive for PCA (overall detection rate of 45.5\%): 6 PCA were detected by target biopsy and 4 cancer by random biopsy. Significant prostate cancer (defined as the presence of Gleason pattern 4) was detected in 4/10 patients (Significant PCA detection rate of $40 \%$ ) and all significant PCA were detected by MRI target biopsy. All PCA detected by random biopsy had Gleason score $3+3=6$. In Group B (biopsy naïve patients) 14/37 patients were positive for PCA (overall detection rate of $37.8 \%$ ), Significant prostate cancer was detected in 5/14 patients (Significant PCA detection rate of 35,7\%). No significant side effects were recorded.

Conclusions: Our overall detection rate was $45.5 \%$ and $37.8 \%$ in Group A (patients with previous negative biopsy and persistent suspicion of PCA) and in Group B (biopsy naïve patients) respectively; clinical significant PCA detection rate was respectively $40 \%$ and $35.7 \%$. These results are similar to current literature and promising for the future. We believe that using platforms of co-registered MRI/US fusion biopsy can potentially improve risk stratification and reduces understaging, undergrading and the need for repeat biopsies in biopsy naive patients (using a stereotactic first mapping) and in patients with previous negative biopsy and persistent suspicion of PCA ( using a second MRI/US fusion biopsy).
\end{abstract}

KEY WORDS: MRI/US fusion biopsy; Prostate cancer; Prostate biopsy.

Submitted 15 November 2016; Accepted 15 December 2016

\section{INTRODUCTION}

Transrectal ultrasound-guided random biopsy of the prostate (TRUSB) is still the recommended standard tool for the diagnosis of prostate cancer (PCA) (1). This approach is a blindly sample of the prostate without focus on any specific lesion; there are several limitations in this approach, including failure to detect clinically significant PCA (due to undersampling); imprecise tumor risk stratification (undergrading) and detection of small, low risk clinically insignificant cancers (overdiagnosis) (2). Undersampling can occur in up to $30 \%$ of cases with clinically significant tumors being missed on initial biopsy; undergrading is exstimated at $46 \%$ of cases considered at low risk of progression candidates for active surveillance, based on preoperative systematic biopsy, but with an upgraded Gleason score of 7 or greater at final histopathology (3).

These diagnostic limitations can lead to repeat biopsies (with related side effects and costs), delayed detection of significant PCA and over treatment. Efforts to overcome sampling error include performing multiple repeat biopsies and increasing the core number; this resulted in the overdetection of indolent cancers, morbidity attributed to unnecessary biopsies and an increase in cost.

Several studies have shown that when serial biopsies are indicated, most cancers that are detected are clinically insignificant and the rate of indolent cancer detection increases $(4,5)$. Recently, a 3-D stereotactic mapping of the prostate has been proposed in order to have a threedimensional histological mapping of the gland: this technique can be made by a templated-guided transperineal biopsy that requires anaesthesia (spinal or sedation) and hospitalization, or by a software based transrectal biopsy that records all tracks of the needle on a 3-D map with only a local anaesthesia. This technique should allow not overlapping needle's tracks with a better 3-D volumetric distribution of the biopsies into the prostatic gland and than a better determination of the extent and location of cancer before definitive therapy (6). Advances in imaging have led to the application of magnetic resonance imaging (MRI) for the detection of PCA (7-12) with subsequent development of software-based co-registration allowing for the integration of MRI with real-time TRUS during prostate biopsy. A number of fusion-guided methods and platforms are now commercially available 
with common elements in image and analysis and planning (13-15). We present our initial experience with one of these platforms after the first consecutive 59 patients.

\section{MATERIALS AND METHODS}

Between May 2016 and October 2016, we prospectively evaluated the first consecutive 59 patients who underwent to TRUS biopsy using Trinity Koelis ${ }^{\circledR}$ (Koelis, Grenoble, France) MRI/US Fusion machine. We divided the patients into 2 groups: patients with a previous negative mapping who underwent to a MRI/US fusion re-biopsy (Group A); and biopsy-naïve patients who underwent to a first stereotactic 3-D mapping of the prostate (Group B). MRI images were obtained using a $1.5 \mathrm{~T}$ scanner with a pelvic phased array coil, each suspicious area was further characterized according to the ESUR PI-RADS v.1 global score (12), MRI were done in different hospital without a central review. Two different urologists made all the biopsies. All biopsy core specimens were examined by 2 urogenital pathologists and graded according to the 2005 International Society of Urological Pathology Modified Gleason Grading System (16). Characteristics of the patients (summarized in Table 1) were in Group A: $\mathrm{n}^{\circ}$ of patients $=22$, mean age 64 years $(\mathrm{CI}$ 48-73), mean PSA = $7.7 \mathrm{ng} / \mathrm{ml}$ (CI 4.2- 9.9), mean prostate volume $=55 \mathrm{ml}(\mathrm{CI} 45-82)$, Digital Rectal Examination (DRE) positive ratio $=2 / 22$, number of lesions detected by MRI 1.4 (17 PIRADS 3, 4 PIRADS 4, 1 PIRADS 5), mean cores from each MRI target lesion $=3(\mathrm{CI} 2-5)$, mean total cores $=15$ (CI 12-19). In Group B: $\mathrm{n}^{\circ}$ of patients $=37$, mean age 66 years (CI 49-77), mean PSA $=4.7$ (3.2- 7.9); mean prostate volume $=45 \mathrm{ml}(33-67)$, DRE positive ratio $=5 / 37$, mean total cores $=14$ (CI 10-16)

We standardized our MRI/US fusion biopsy technique using Koelis Trinity in 5 steps:

First step: MRI T2 and/or DWI images are loaded into the Trinity, we manually border the prostate signing the apex, the base the mid gland and additional landmarks of the prostate obtaining a 3-D MRI volume; than we target the suspected areas described in the MRI report in a semiautomatic process. This step is usually done the day before procedure

\section{Table 1.}

Characteristics of the patients.

Group A: patients with a previous negative mapping who underwent to a MRI/US fusion re-biopsy.

Group B: biopsy-naïve patients who underwent to a first stereotactic 3-D mapping of the prostate.

\begin{tabular}{|lcc|}
\hline & Group A & Group B \\
\hline Number of patients & 22 & 37 \\
\hline Mean age & 64 & 66 \\
\hline Mean PSA & 7.7 & 4.7 \\
\hline DRE positive & $2 / 22$ & $5 / 37$ \\
\hline Mean Prostate Volume & 55 & 46 \\
\hline Total cores & 15 & 14 \\
Mean core from each target & 3 & \\
\hline PIRADS 3 & $17 / 22$ & \\
\hline PIRADS 4 & $4 / 22$ & \\
\hline PIRADS 5 & $1 / 22$ & \\
\hline
\end{tabular}

Second step: a 3D TRUS volume is obtained by a real-time TRUS examination in 3 planes (transversal, 60 degree longitudinal turning the probe on the right and on left) by an end fire probe with a rotating 360 degree head. We border the prostate volume in a similar way to MRI process.

Third step: automatic elastic fusion of the MRI and ultrasound volumes is done by the machine's software pressing a button. We check the correct fusion of the volumes

Fourth step: virtual simulation of bioptical tracking with a visual feedback on the fused target volume is done Fifth step: if the virtual simulation of the track is inside the target lesion, we press the button of the needle and a real biopsy is done followed by 3D-TRUS acquisition of the real track with the needle still in the gland. A mean of 2 cores were obtained from each MRI-target area. Then at least a 12-core random biopsy were performed in all patients.

In case of stereotactic first round biopsy in biopsy naive patient (Group B) step 1 and 3 are avoided, we recorded all real track in a 3-D TRUS map.

\section{RESULTS}

Our results are summarized below and displayed in Table 2.

Group A (22 patients): 10/22 positive for PCA (overall detection rate of 45.5\%). 6 PCA were detected by target biopsy and 4 cancer by random biopsy. Significant prostate cancer (defined as the presence of Gleason pattern 4) was detected in 4/10 patients (significant PCA detection rate of $40 \%$ ), all significant PCA were detected in MRI target biopsy (1/17 PIRADS 3, 2/4 PIRADS 4, 1/1 PIRADS 5). All PCA detected by random biopsy had Gleason score $3+3=6$.

Group B (37 biopsy naïve patients): 14/37 positive for PCA (overall detection rate of $37.8 \%$ ), Significant prostate cancer was detected in 5/14 patients (Significant PCA detection rate of $35,7 \%$ ).

The mean time of the procedure was $42 \mathrm{~min}$ (C.I. 22-55) in the initial 10 patients and $27 \mathrm{~min}$ in the following 10 patients (C.I. 19-35) in Group A, 27 min (C.I. 19-45) in the initial 10 patients and $17 \mathrm{~min}$ in the following 10 patients (C.I. 10-25) in Group B.

No significant side effects were recorded in either group (such as fever, urinary retention, urosepsis, and hospitalization).

\section{Table 2.}

Results.

\begin{tabular}{lcc}
\hline & Group A & Group B \\
\hline Overall PCA detection rate & $10 / 22(45.5 \%)$ & $14 / 37(37.8 \%)$ \\
\hline Significant PCA detection rate & $4 / 10(40 \%)$ & $5 / 14(35.7 \%)$ \\
\hline PCA in target lesion & $6 / 10$ & \\
\hline PCA in random biopsy core & $4 / 10$ \\
\hline Significant PCA detected in target lesion & $4 / 6$ \\
\hline PIRADS 3 & $1 / 6$ \\
\hline PIRADS 4 & $2 / 6$ \\
\hline PIRADS 5 & $1 / 6$ \\
\hline Significant PCA in random biopsy core & $0 / 4$
\end{tabular}




\section{Discussion}

We considered many commercial platforms of co-registered MRI/US fusion biopsy devices commercially available. These devices vary by method of co-registration (mechanical, electromagnetic or real-time) and use a different hardware platform to align the biopsy with the co-registered image. We chose Trinity Koelis because we considered this option to be the best compromise between accuracy, reproducibility and feasibility in our daily practice.

A recent systematic review shows that MRI-TRUS image fusion targeted biopsies detect more clinically significant cancers compared with standard biopsy techniques: the median detection rate of any cancer was $43.4 \%$ and $50.5 \%$ in the standard biopsy strategy versus MRI-TRUS image fusion biopsy; the median detection of clinically significant disease was $23.6 \%$ (range: $4.8-52 \%$ ) for standard biopsy and 33.3\% (range: $13.2-50 \%$ ) for MRITRUS image fusion targeted biopsy However, patient populations differ quite a bit between the different studies regarding the amount of patients with a previous negative biopsy or patients that were biopsy naive (17). Our overall detection rate was $45.5 \%$ and $37.8 \%$ in MRI-US fusion biopsy (patients with previous negative biopsy and persistent suspicion of PCA) and in stereotactic biopsy (biopsy naïve patients); clinical significant PCA detection rate was respectively $40 \%$ and $35.7 \%$. These results are similar to those reported in the current literature and promising for the future. The main limitations of our study were the small number of patients, difference in expertise of the radiologists, our learning curve of at least the first 10 cases.

Until now our main problems using this technique are the following: it is not easy to border the target lesion without radiologist's help if it is visible only in DWI sequence; because this is a freehand procedure (operator dependent technique) without a probe stepper, even a little movement of the probe in the passage from virtual track simulation to real biopsy could miss the target lesion (especially if the diameter is $<1 \mathrm{~cm}$ ).

\section{Conclusions}

MRI/US fusion biopsy in patients with a previous negative mapping but persistent suspicion of PCA represents a useful tool to address many of the limitations of contemporary systematic re-biopsy (reduce false-negatives, improve risk classification, contribute to the reduction of repeat biopsies and overdetection). Among men with no previous biopsy its role is poorly defined, but we believe that at least a stereotactic first bioptical mapping (recording all bioptical tracks in a 3-D map) can not only increase cancer detection rate but, if a re-biopsy will be necessary, a MRI-US fusion biopsy can be done using the previous recorded 3-D map, avoiding the same tracks of first mapping and improving the results of this technique.We believe that among men with suspicion of PCA, stereotactic first mapping and MRI/US fusion re- biopsy potentially improves risk stratification and reduces understaging, undergrading and the need for repeat biopsies. The optimal method for MR targeted biopsy has not yet been established; further comparative studies with standard of practice and evaluation of cost-effectiveness are warranted.

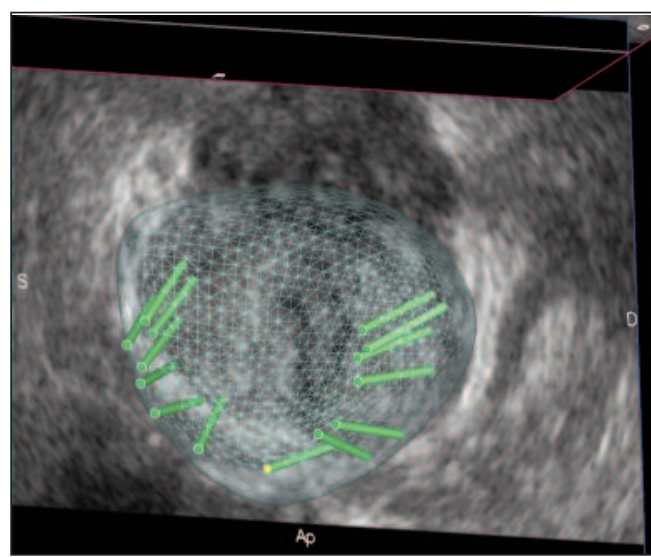

Figure 1.

First

stereotactic

3-D mapping

of the

prostate

in a biopsy-

naive patient.

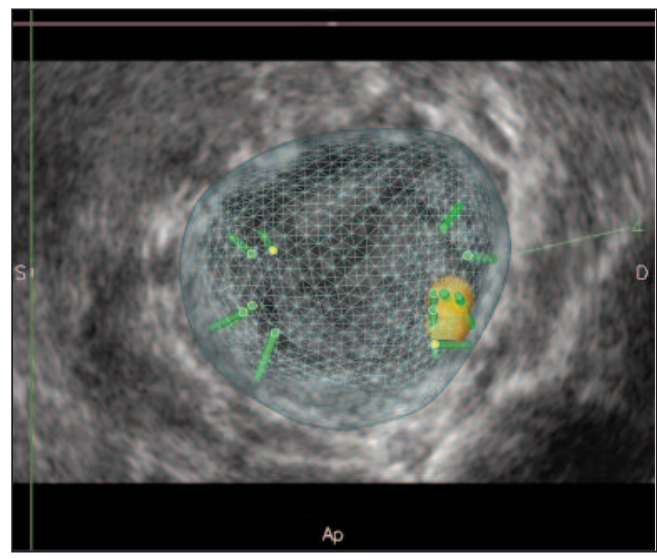

Figure 2.

MRI/US fusion

re-biopsy in a patients with a previous negative mapping (4 target cores from a MRI lesion with a PIRADS score 3 at right apex plus 9 random cores).

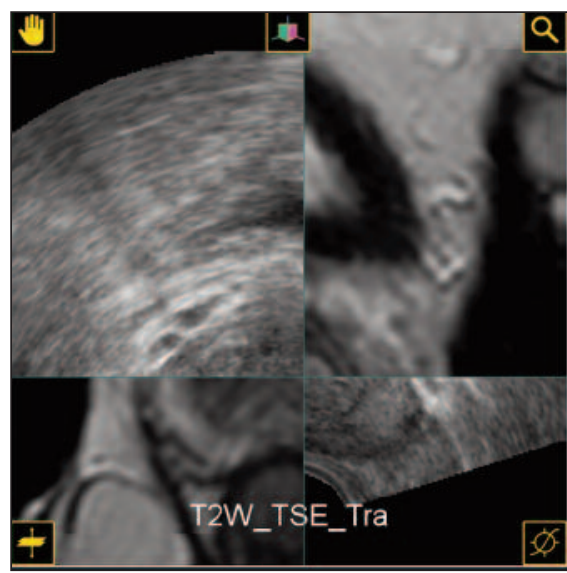

Figure 3.

Automatic elastic fusion of the MRI and ultrasound volumes is done by the machine's software.

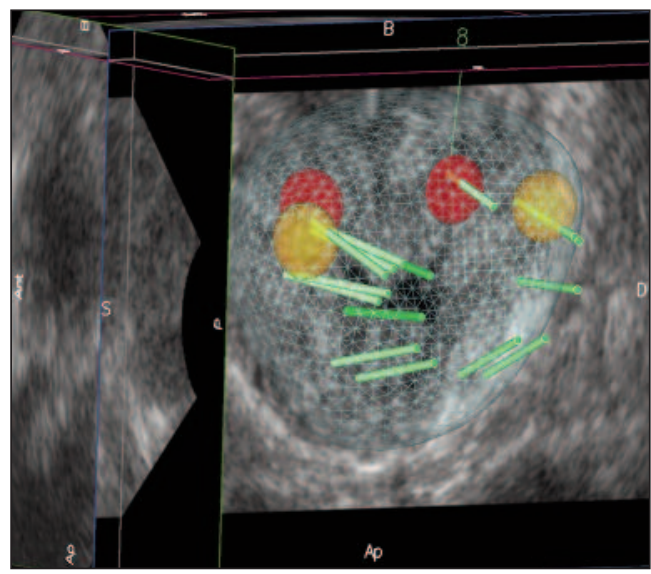

Figure 4. MRI/US fusion target and random re-biopsy: 2 MRI lesions with a PIRADS score of 3 (orange) and $2 \mathrm{MRI}$ lesions with PIRADS 4 (red). 


\section{REFERENCES}

1. Bjurlin MA, Carter HB, Schellhammer P, et al. Optimization of initial prostate biopsy in clinical practice: sampling, labeling and specimen processing. J Urol. 2013; 189:2039.

2. Serefoglu EC, Altinova S, Ugras NS, et al. How reliable is 12-core prostate biopsy procedure in the detection of prostate cancer? Can Urol Assoc J. 2013; 7:E293-E298

3. Mufarrij P, Sankin A, Godoy G, et al. Pathologic outcomes of candidates for active surveillance undergoing radical prostatectomy. Urology. 2010; 76:689.

4. Walz J, et al. High incidence of prostate cancer detected by saturation biopsy after previous negative biopsy series. Eur Urol. 2006; 50:498.

5. Eichler K, et al. Diagnostic value of systematic biopsy methods in the investigation of prostate cancer: a systematic review. J Urol. 2006; 175:1605.

6. Moran BJ, et al. Re-biopsy of the prostate using a stereotactic transperineal technique. J Urol. 2006; 176:1376.

7. Futterer JJ, et al. Can clinically significant prostate cancer be detected with multiparametric magnetic resonance Imaging? A systematic review of the literature. Eur Urol. 2015; 68:1045.

8. Schoots IG, et al. Magnetic resonance imaging-targeted biopsy may enhance the diagnostic accuracy of significant prostate cancer detection compared to standard transrectal ultrasound-guided biopsy: a systematic review and meta-analysis. Eur Urol. 2015; 68:438.

9. Panebianco V, et al. Multiparametric magnetic resonance imaging vs. standard care in men being evaluated for prostate cancer: a randomized study. Urol Oncol. 2015; 33:17 e1.
10. Barentsz JO, Weinreb JC, Verma S, et al. Synopsis of the PIRADS $v 2$ guidelines for multiparametric prostate magnetic resonance imaging and recommendations for use. Eur Urol. 2016; 69:41-49.

11. Vache $T$, et al. Characterization of prostate lesions as benign or malignant at multiparametric MR imaging: comparison of three scoring systems in patients treated with radical prostatectomy. Radiology. 2014;. 272:446.

12. Barentsz JO, et al. ESUR prostate MR guidelines 2012. Eur Radiol. 2012; 22:746.

13. Moore CM, Robertson NL, Arsanious N, et al. Image-guided prostate biopsy using magnetic resonance imaging-derived targets: a systematic review. Eur Urol. 2013; 63:125.

14. Van Hove A, et al. Comparison of image-guided targeted biopsies versus systematic randomized biopsies in the detection of prostate cancer: a systematic literature review of well-designed studies. World J Urol. 2014; 32:847.

15. Siddiqui MM, et al. Comparison of MR/ultrasound fusion-guided biopsy with ultrasound-guided biopsy for the diagnosis of prostate cancer. JAMA. 2015; 313:390.

16. Epstein JI, Allsbrook WC, Amin MB, Egevad LL, Grading ISUP, ISUP Grading Committee The 2005 International Society of Urological Pathology (ISUP) consensus conference on Gleason grading of prostatic carcinoma. Am J Surg Pathol. 2005; 29:1228-1242.

17. Valerio M, Donaldson I, Emberton M, et al. Detection of Clinically Significant Prostate Cancer Using Magnetic Resonance ImagingUltrasound Fusion Targeted Biopsy: A Systematic Review. Eur Urol. 2015; 68:8-19.

\author{
Correspondence \\ Vito Lacetera, MD, Urologist \\ Vito.Lacetera@gmail.com \\ Bernardo Cervelli, MD, Urologist \\ Bernardo.Cervelli@ospedalimarchenord.it \\ Antonio Cicetti, MD, Urologist \\ Antonio.Cicetti@ospedalimarchenord.it \\ Giuliana Gabrielloni, MD, Urologist \\ Giuliana.Gabrielloni@ospedalimarchenord.it \\ Gianluca Giglioni, MD \\ Gianluca.Giglioni@ospedalimarchenord.it - piallu88@gmail.com \\ Michele Montesi, MD, Urologist \\ Michele.Montesi@ospedalimarchenord.it \\ Roberto Morcellini, MD, Urologist \\ Roberto.Morcellini@ospedalimarchenord.it \\ Gianni Parri, MD, Urologist \\ Gianni.Parri@ospedalimarchenord.it \\ Emilio Recanatini, MD, Urologist \\ Emilio.Recanatini@ospedalimarchenord.it \\ Valerio Beatrici, MD, Urologist \\ Valerio.Beatrici@ospedalimarchenord.it \\ Azienda Ospedaliera Ospedali Riuniti Marche Nord \\ Piazzale Cinelli 4, 61121 Pesaro, Italy \\ Andrea Benedetto Galosi, MD, Associate Professor of Urology \\ galosiab@yahoo.it \\ Clinica Urologica, Università Politecnica delle Marche, \\ Azienda Ospedaliera Ospedali Runiti, Ancona, Italy
}

